\title{
Momentum distributions of particles from three-body halo fragmentation: Final state interactions
}

\author{
E. Garrido, D. V. Fedorov, and A. S. Jensen \\ Institute of Physics and Astronomy, Aarhus University, DK-8000 Aarhus C, Denmark
}

(Received 17 October 1995)

\begin{abstract}
Momentum distributions of particles from nuclear breakup of fast three-body halos are calculated consistently, and applied to ${ }^{11} \mathrm{Li}$. The same two-body interactions between the three particles are used to calculate the ground state structure and the final state of the reaction processes. We reproduce the available momentum distributions from ${ }^{11} \mathrm{Li}$ fragmentation, together with the size and energy of ${ }^{11} \mathrm{Li}$, with a neutron-core relative state containing a $p$-state admixture of 20\%-30\%. The available fragmentation data strongly suggest an $s$ state in ${ }^{10} \mathrm{Li}$ at about $50 \mathrm{keV}$ and indicate a $p$ state around $500 \mathrm{keV}$. [S0556-2813(96)00906-5]

PACS number(s): 25.60.-t, 21.45.+v, 21.60.Gx, 27.20.+n
\end{abstract}

The existence of halo nuclei is by now well established as weakly bound and spatially extended systems [1]. The dominant features of these unusual nuclei are most easily described by few-body models. The many degrees of freedom are then divided into the approximately frozen (core) and the active (halo) degrees of freedom. Substantial efforts have been allocated to investigations of three-body halos (one core and two halo particles) and in particular to bound threebody systems where all two-particle subsystems are unbound [2]. The nuclear prototypes of these so-called Borromean systems are ${ }^{6} \mathrm{He}\left({ }^{4} \mathrm{He}+n+n\right)$ and ${ }^{11} \mathrm{Li}\left({ }^{9} \mathrm{Li}+n+n\right)$, both thoroughly discussed in a general framework in [3].

The most detailed source of experimental information about the structure of these nuclei is the momentum distributions of the "particles" resulting from fragmentation reactions [4-7]. One major problem in the interpretation of such measurements is the inherent mixture of effects from the original structure and the reaction mechanism [8]. Both should therefore be consistently incorporated in model calculations.

The simplest model assumes the sudden approximation, where one of the particles is instantaneously removed from the three-body system while the other two particles remain completely undisturbed. Clearly this can only be justified for reaction times much shorter than the characteristic time for the motion of the three particles in the system. Since halo nuclei are weakly bound and the beam energy is very high, this requirement is exceedingly well fulfilled. The observed momentum distributions therefore seem to provide direct information about the three-body wave function [9]. However, the interactions between the remaining two particles are often essential especially when low lying resonances are present $[5,10,11]$. In principle, processes in which the three particles move along together after the fragmentation are also possible. However, they occur through Coulomb dissociation, requiring a heavy target, or by use of a low energy beam. Since we consider a light target and a high energy beam, this kind of reaction is then out of our model, and they are not considered in this paper.

The final state two-body interactions (FSI's) are active in the last part of the reaction process and they determine simultaneously the three-body structure of the initial halo nucleus. A consistent treatment is necessary to allow reliable interpretations of this major source of detailed experimental information. In this paper we report on such model calculations, where we combine an accurate three-body description with an equally accurate computation of momentum distributions. After a general discussion we shall present detailed calculations of momentum distributions of particles from fragmentation reactions of ${ }^{11} \mathrm{Li}$ when FSI's are included.

After the collision in which one particle is suddenly removed, the probability of finding the remaining two particles with relative momentum $\mathbf{k}_{x}$ and total momentum $\mathbf{k}_{y}$ relative to the center of mass of the three-body system is proportional to the overlap

$$
\Psi\left(\mathbf{k}_{x}, \mathbf{k}_{y}\right)=\left\langle e^{i \mathbf{k}_{y} \cdot \mathbf{y}} e^{i \mathbf{k}_{x} \cdot \mathbf{x}} \mid \Psi(\mathbf{x}, \mathbf{y})\right\rangle,
$$

where $\Psi(\mathbf{x}, \mathbf{y})$ is the three-body wave function. The coordinates $\mathbf{x}$ and $\mathbf{y}$ are the usual Jacobi coordinates [2,3] where $\mathbf{x}$ is drawn between the two particles surviving after the fragmentation. Without FSI's the momentum distribution or the differential cross section is then proportional to the square of the Fourier transform of the three-body wave function.

Inclusion of FSI's now amounts to substituting the plane wave $e^{i \mathbf{k}_{x} \cdot \mathbf{x}}$ in Eq. (1) by the appropriate distorted two-body wave function $w$. The momentum distribution is then given by

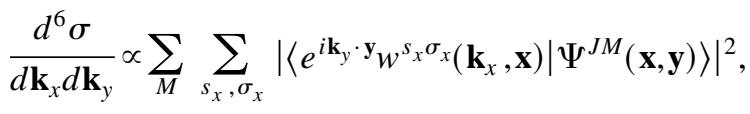

where $J$ is the total spin of the halo nucleus, and $s_{x}$ and $\sigma_{x}$ are the spin of the two-body final state and its projection. The summations in Eq. (2) arise from the average over initial states $(M)$ and the sum over final states $\left(s_{x}\right.$ and $\left.\sigma_{x}\right)$.

In our calculation the three-body wave function is obtained by solving the Faddeev equations in coordinate space, where the nucleon-nucleon potential is fitted to low energy $s$ - and $p$-wave nucleon-nucleon scattering data, and the neutron-core potential is adjusted to give the proper binding energy and mean square radius of the three-body system $[12,13]$.

The partial wave expansion of the two-body final state wave function is written as [14] 


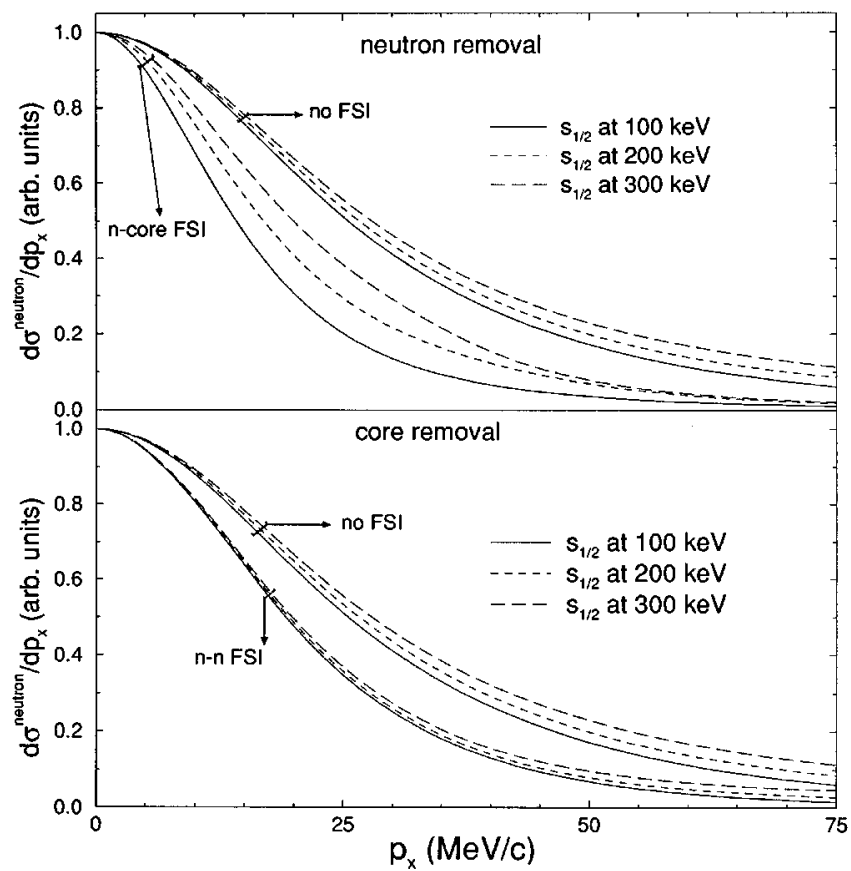

FIG. 1. Upper part: the neutron momentum distributions from neutron removal process in ${ }^{11} \mathrm{Li}$ fragmentation. $p_{x}$ is the $x$ Cartesian component of the momentum of the neutron relative to the center of mass of the three-body system. The $s_{1 / 2}$ virtual state is placed at 100 $\mathrm{keV}$ (solid line), $200 \mathrm{keV}$ (dashed line), and $300 \mathrm{keV}$ (long dashed line), respectively. The upper set of curves corresponds to calculations without FSI's, while FSI's are included in the lower set. Lower part: the same as before for core breakup reactions.

$$
\begin{aligned}
w^{s \sigma}(\mathbf{k}, \mathbf{x})= & \sqrt{\frac{2}{\pi} \frac{1}{k x}} \sum_{j, l, m} u_{l s}^{j}(k, x) \mathcal{Y}_{j l s}^{m^{*}}\left(\Omega_{x}\right) \\
& \times \sum_{m_{l}=-l}^{l}\left\langle l s ; m_{l} \sigma \mid j m\right\rangle i^{l} Y_{l m_{l}}\left(\Omega_{k}\right),
\end{aligned}
$$

where the radial functions $u_{l s}^{j}(k, x)$ are obtained by solving the Schrödinger equation with the appropriate two-body potential. Finally, we calculate the expression in Eq. (2) and subsequently integrate over the unobserved quantities to obtain the measured momentum distributions. The details of the formalism will be presented elsewhere.
We reveal the general results of the procedure described above by using the three-body halo ${ }^{11} \mathrm{Li}\left({ }^{9} \mathrm{Li}+n+n\right)$ as an example, and neglecting the spin of the core. The neutronneutron interaction is fitted as previously indicated, and the neutron-core $s$ - and $p$-wave potentials are varied to study the dependence of the momentum distribution on the positions of the resonances in ${ }^{10} \mathrm{Li}$, as well as the effect of the inclusion of FSI's. The binding energy and mean square radius of ${ }^{11} \mathrm{Li}$ are kept fixed to the experimental values.

In the upper part of Fig. 1 we show the resulting neutron momentum distributions after one of the neutrons is removed by the target for three different energies of the $s_{1 / 2}$ virtual state $(100,200$, and $300 \mathrm{keV})$. The upper set of curves is obtained without FSI's. They show a little variation, since they depend mainly upon the binding energy and rms radius of ${ }^{11} \mathrm{Li}$. The final state interactions substantially reduce the widths, the stronger the lower the virtual state is. In Table I we summarize the full width at half maximum (FWHM) for the curves in Fig. 1. Here we also give the FWHM of the core one-dimensional momentum distributions, which are much less influenced by FSI's due to the larger mass.

The lower part of Fig. 1 shows the same kind of calculation for core breakup reactions, where the core is destroyed during the interaction, keeping the validity of the sudden approximation. The interest in this kind of reaction is that now the final state interaction (neutron-neutron interaction) is well known, reducing the uncertainties involved in the calculation. We first observe that variation of the position of the $s_{1 / 2}$ virtual state only produces a small change in the momentum distributions. That is expected, since the final state neutron-neutron interaction is independent of the properties of ${ }^{10} \mathrm{Li}(n+$ core $)$. However, when comparing with the results without FSI's, as shown by the upper set of curves, we still observe a noticeable variation (note that the curves without FSI's are identical to the corresponding curves in the upper part of the figure). Now the FWHM of the distribution is around $40 \mathrm{MeV} / c$, broader than the distributions obtained when one of the neutrons is removed, but narrower than the calculation without FSI's. As seen from Table I, the agreement with the experimental data is quite good, especially for low energies of the $s$ virtual state. This is in complete agreement with the low lying $s$ virtual state $(\sim 50 \mathrm{keV})$ recently suggested in [5] along with a $p$-state resonance near 0.5 $\mathrm{MeV}$.

TABLE I. Comparison of the full width at half maximum in $\mathrm{MeV} / c$ of the longitudinal momentum distributions from ${ }^{11} \mathrm{Li}$ fragmentation for different positions of the $s_{1 / 2}$ virtual state. Columns $2-4$ refer to neutron momentum distributions, while 5 and 6 refer to core momentum distributions. $(n-c)$ FSI's between ${ }^{9} \mathrm{Li}$ and the neutron have been included (neutron removal process). ( $\left.n-n\right)$ FSI's between the two neutrons have been included (core breakup process).

\begin{tabular}{lccccc}
\hline \hline & \multicolumn{3}{c}{ Neutron } & \multicolumn{2}{c}{ Core } \\
& no FSI's & FSI $^{(n-c)}$ & FSI $^{(n-n)}$ & no FSI's & FSI $^{(n-c)}$ \\
\hline$E_{s_{1 / 2}}=100 \mathrm{keV}$ & 51.1 & 28.3 & 38.4 & 68.5 & 55.1 \\
$E_{s_{1 / 2}}=200 \mathrm{keV}$ & 53.2 & 33.9 & 39.0 & 69.8 & 58.0 \\
$E_{s_{1 / 2}}=300 \mathrm{keV}$ & 55.8 & 39.9 & 39.7 & 71.4 & 60.4 \\
Experimental & & $25-35^{\mathrm{a}}$ & $43 \pm 3^{\mathrm{b}}$ & & $49 \pm 3^{\mathrm{c}}$ \\
\hline \hline
\end{tabular}

${ }^{\mathrm{a}}$ See, for instance, [4].

${ }^{\mathrm{b}}$ Data from [6].

${ }^{\mathrm{c}}$ Data from [7]. 


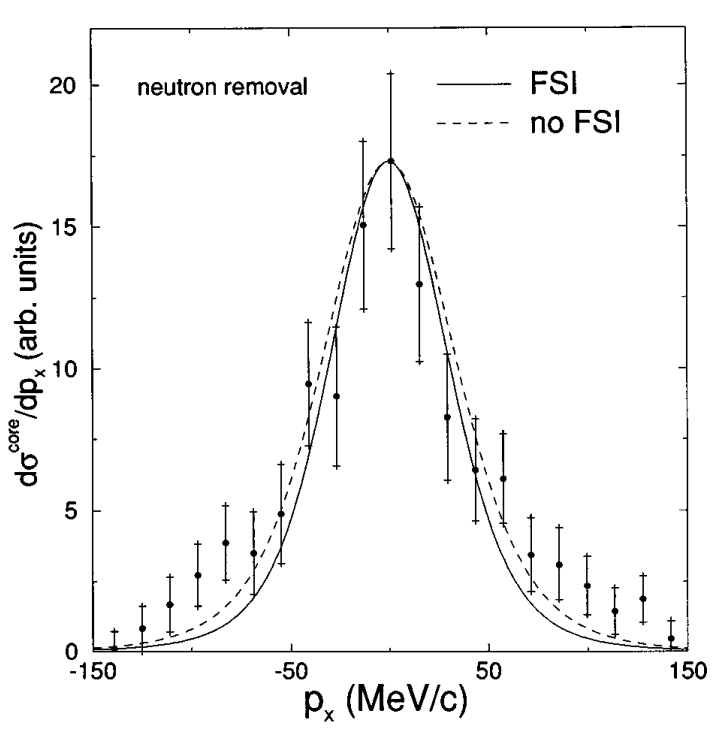

FIG. 2. Transverse core momentum distribution after neutron removal from ${ }^{11} \mathrm{Li}$. $p_{x}$ is the $x$ Cartesian component of the momentum of the core relative to the center of mass of the three-body system. The solid (dashed) line is the calculation with (without) FSI's. Data from [7].

It should be mentioned that a narrow momentum distribution can also be obtained with a low lying $p$ resonance. However in this case the lowest $s$ state must be shifted towards much higher energies to reproduce the correct binding energy of ${ }^{11} \mathrm{Li}$.

Up to now, we have neglected the spin dependence of the neutron-core interaction or equivalently assumed that the spin of the core is zero. Of course, this is not realistic (the spin of ${ }^{9} \mathrm{Li}$ is $3 / 2$ ), and the neutron-core interaction should include a spin dependence, splitting the two possible $s$ states of ${ }^{10} \mathrm{Li}$ with total angular momentum 1 and 2 . To do this, we have in the neutron-core potential included one term proportional to $\mathbf{s}_{n} \cdot \mathbf{s}_{c}$, where $\mathbf{s}_{n}$ is the spin of the neutron and $\mathbf{s}_{c}$ the spin of the core [13]. For simplicity, the spin splitting term has been introduced only in the $s$ wave.

It is now possible to place an $s$ state at $50 \mathrm{keV}$ and the lowest $p$ resonance at $500 \mathrm{keV}$, as suggested by the analyses in [5]. Simultaneously we are able to vary the content of $s$ and $p$ waves in the neutron-core subsystem of ${ }^{11} \mathrm{Li}$. Such realistic calculations can be compared directly with the experimental data. We then place the lowest $p$ resonance at 500 $\mathrm{keV}$, and the lowest $s$ state with total angular momentum 2 at $50 \mathrm{keV}$. Then we use the spin-orbit potential in the neutroncore interaction to vary the total $p$-state content in the ${ }^{11} \mathrm{Li}$ wave function, and finally we adjust the energy of the second $s$ state (with total angular momentum 1) to recover the correct binding energy and mean square radius in the ${ }^{11} \mathrm{Li}$. This procedure determines completely the low lying resonance structure of ${ }^{10} \mathrm{Li}$. Very similar results are obtained by placing the $s$ state with angular momentum 1 at $50 \mathrm{keV}$, and instead adjusting the energy of the $s$ state with angular momentum 2 .

In Fig. 2 we show the one-dimensional transverse momentum distribution of ${ }^{9} \mathrm{Li}$ fragments. The experimental data [7] correspond to a reaction at $280 \mathrm{MeV} /$ nucleon in a $\mathrm{C}$ target. The ${ }^{11} \mathrm{Li}$ wave function contains around $26 \%$ of $p$ wave in the neutron-core subsystem, but the result is almost

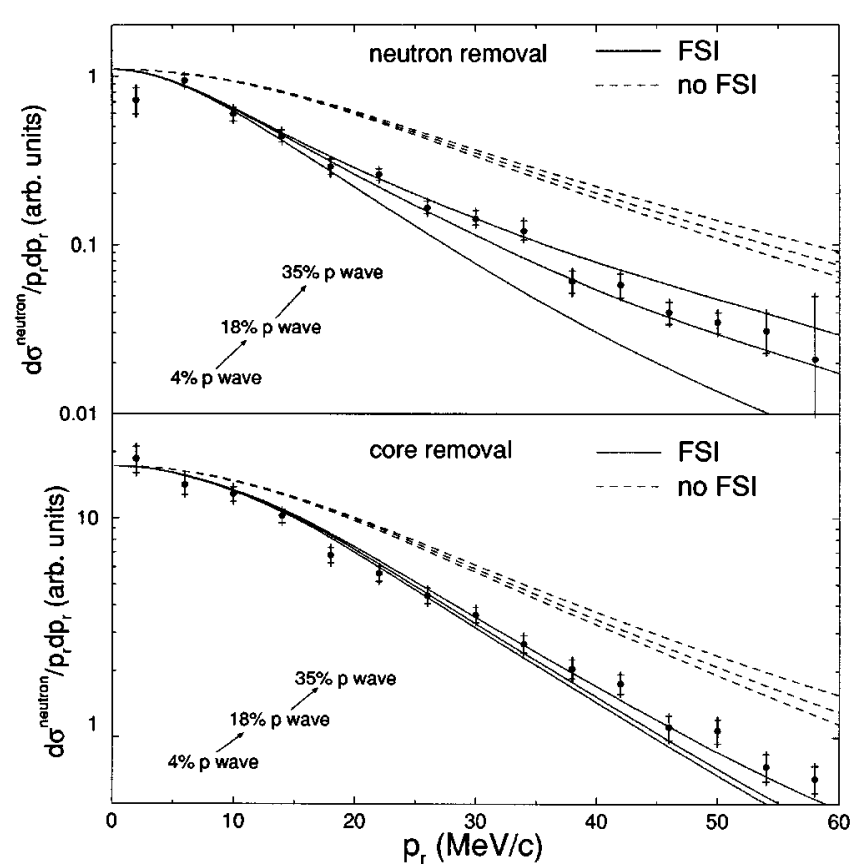

FIG. 3. Radial neutron momentum distribution for neutron removal (upper part) and core breakup (lower part) reactions of ${ }^{11} \mathrm{Li} . p_{r}=\sqrt{p_{x}^{2}+p_{y}^{2}}$ is the cylindrical radial component of the momentum of the neutron relative to the center of mass of the threebody system. Results with (solid line) and without (dashed line) FSI's are presented. The $p$-wave content in the neutron- ${ }^{9} \mathrm{Li}$ subsystem (from narrower to broader distributions) is $4 \%, 18 \%$, and $35 \%$. Experimental data are taken from [6] and [5].

independent of the $p$-state admixture. The results of the computation with (solid line) and without (dashed line) FSI's are shown in the figure. The curves have been convoluted with the experimental beam profile [7]. As expected, the effect of FSI's is rather small in the central region. For momenta larger than around $75 \mathrm{MeV} / c$ the neutrons are inside the core, and our three-body model is not valid anymore. Also, in the high momentum region other effects, like diffraction processes, should be considered. The good agreement in the central region shows that our ${ }^{11} \mathrm{Li}$ wave function is accurate enough to describe the process.

To evaluate the effect of FSI's on the momentum distributions we investigate neutron distributions, which are much more sensitive to them. The results are shown in Fig. 3 for the two-dimensional neutron momentum distribution, both for neutron removal processes (upper part) and for core breakup reactions (lower part). The appropriate FSI's are included, i.e., neutron-core in the first case and neutronneutron in the second case. In both cases the experimental data correspond to reactions of ${ }^{11} \mathrm{Li}$ at $280 \mathrm{MeV} /$ nucleon with a $\mathrm{C}$ target $[5,6]$. Several ${ }^{11} \mathrm{Li}$ wave functions with different content of $s$ and $p$ waves in the neutron-core channel have been used. In particular, going from the narrower to the broader distributions, the curves correspond to calculations with $4 \%, 18 \%$, and $35 \%$ of the $p$ wave. In both parts of the figure, FSI's are essential to recover the observed behavior of the distributions.

The core breakup process (lower part) is almost insensitive to the structure of ${ }^{11} \mathrm{Li}$ and the peculiarities of the neutron-core interaction. This is due to the fact that the final state interactions between the two neutrons are independent 
of the neutron-core potential. The nice agreement with the experiment strongly supports the method and the model. In the upper part of Fig. 3 the two-dimensional neutron momentum distribution for neutron removal reactions are shown. Now we see that a ${ }^{11} \mathrm{Li}$ wave function with a small content of the $p$ wave clearly underestimates the width of the momentum distribution, while a wave function with more than $35 \%$ of the $p$ wave in the neutron- ${ }^{9} \mathrm{Li}$ subsystem overestimates the width. The best results are obtained when a $p$-wave admixture of around $26 \%$ is used. The data in the upper part of Fig. 3 also support the choice of a $500 \mathrm{keV} \mathrm{p}$ state, since this energy corresponds to the bump observed around $p_{r} \simeq 30 \mathrm{MeV} / c$.

We have calculated momentum distributions of fragments from high energy nuclear breakup reactions of three-body Borromean halo nuclei. The two-body interaction in the three-body description of the halo nucleus is identical to the final state interaction between the two particles remaining after the target has removed the third particle. We maintain this consistency in the description. The final state interaction may significantly reduce the width of the momentum distribution of the light particle. The size of this reduction strongly depends on the resonance structure of the remaining two-body system.

After extraction of the general properties, we applied the model to fragmentation of the three-body halo nucleus

${ }^{11} \mathrm{Li}$. First, we compared the core-momentum distribution after neutron removal with experimental data. The results are only marginally sensitive to final state interactions and the sudden approximation only recovers the ground state wave function in momentum space. The good agreement with measurements therefore demonstrates the validity of our ${ }^{11} \mathrm{Li}$ three-body model and the reaction mechanism assumed.

Second, we computed two-dimensional neutron distributions after core breakup. They are rather insensitive to the characteristics of the neutron-core interaction which only enters through the properties of the ground state wave function. However, the final state neutron-neutron interaction is essential to reproduce the measured data. This demonstrates the validity of our method of including final state interactions.

Third, we computed two-dimensional neutron distributions after one-neutron removal. They depend crucially on the neutron-core interaction which now enters both through the properties of the ground state wave function and through the final state interaction. The structure of ${ }^{11} \mathrm{Li}$ must then contain a $p$-state admixture of about $26 \%$ in the relative neutron-core system. The same interaction determines the structure of ${ }^{10} \mathrm{Li}$, where a $p$-state resonance at about $500 \mathrm{keV}$ produces the small shoulder at about $30 \mathrm{MeV} / c$ in the experimental distribution. The statistical average of the two spin-split $s$ virtual states must then be at about $700 \mathrm{keV}$ in order to reproduce the binding energy of ${ }^{11} \mathrm{Li}$. Furthermore, the narrow momentum distribution can only be reproduced with the lowest $s$ virtual state in ${ }^{10} \mathrm{Li}$ at about $50 \mathrm{keV}$.

One of us (E.G.) acknowledges support from the European Union through the Human Capital and Mobility program Contract No. ERBCHBGCT930320.
[1] P.G. Hansen, A.S. Jensen, and B. Jonson, Annu. Rev. Nucl. Part. Sci. 45, 591 (1995)

[2] D.V. Fedorov, A.S. Jensen, and K. Riisager, Phys. Rev. C 49, 201 (1994).

[3] M.V. Zhukov, B.V. Danilin, D.V. Fedorov, J.M. Bang, I.S. Thompson, and J.S. Vaagen, Phys. Rep. 231, 151 (1993).

[4] R. Anne et al., Phys. Lett. B 250, 19 (1990).

[5] M. Zinser et al., Phys. Rev. Lett. 75, 1719 (1995).

[6] T. Nilsson et al., Europhys. Lett. 30, 19 (1995).

[7] F. Humbert et al., Phys. Lett. B 347, 198 (1995).

[8] C.A. Bertulani, L.F. Canto, and M.S. Hussein, Phys. Rep. 226, 281 (1993).
[9] I.S. Thompson and M.V. Zhukov, Phys. Rev. C 49, 1904 (1994).

[10] F. Barranco, E. Vigezzi, and R.A. Broglia, Phys. Lett. B 319 387 (1993).

[11] A.A. Korsheninnikov and T. Kobayashi, Nucl. Phys. A567, 97 (1994).

[12] D.V. Fedorov, A.S. Jensen, and K. Riisager, Phys. Rev. C 50, 2372 (1994).

[13] D.V. Fedorov, E. Garrido, and A.S. Jensen, Phys. Rev. C 51, 3052 (1995).

[14] R.G. Newton, Scattering Theory of Waves and Particles, 2nd ed. (Springer-Verlag, New York, 1982), p. 444. 